\title{
OPTOELECTRONIC PROPERTIES OF (001) AND (111) LATTICE-MATCHED AND STRAINED QUANTUM WIRE LASERS-COMPARISON WITH QUANTUM WELL LASERS
}

\author{
IGOR VURGAFTMAN and JASPRIT SINGH \\ Solid State Electronics Laboratory, Department of Electrical Engineering and Computer Science, \\ The University of Michigan, Ann Arbor, MI 48109-2122, U.S.A.
}

\begin{abstract}
We investigate the optoelectronic properties of GaAs wires along the [001] and [111] directions for a range of cross sections and compare these with the corresponding parameters for optimized quantum wells. The relative benefits of built-in strain in quantum wire and quantum well systems are also studied. We find that the threshold current density in the $100 \times 50 \AA$ wire is $80 \mathrm{~A} / \mathrm{cm}^{2}$ compared with $560 \mathrm{~A} / \mathrm{cm}^{2}$ in a $50 \AA$ GaAs well. The differential gain in quantum wires is increased by an order of magnitude in comparison with quantum wells, and can be as high as $10^{-13} \mathrm{~cm}^{2}$. A narrow gain spectrum is calculated for quantum wire lasers ensuring high mode selectivity and strong damping of relaxation oscillations. We also consider the issue of carrier relaxation in quantum-confined structures, which may impose an upper limit on the laser modulation frequency. Performing a Monte Carlo simulation of the relaxation process, we find that the electron relaxation time in quantum wires is increased to above $100 \mathrm{ps}$ in comparison with $\approx 10 \mathrm{ps}$ in quantum wells. Within our model, this unusually slow relaxation process may limit the small-signal modulation frequency of quantum wires to several $\mathbf{G H z}$. Since modulation frequencies of over $30 \mathrm{GHz}$ have been achieved in quantum wells, we conclude that although quantum wires possess superior optoelectronic properties as compared with quantum wells, the degraded small-signal response can make use of quantum wire lasers for high speed applications undesirable.
\end{abstract}

The rapid development of the quantum well laser technology is the direct consequence of the advantages that the 2D step-like density of states offers over the monotonically increasing $3 \mathrm{D}$ density of states in the conventional double heterostructure laser[1-3]. However, the 2D density of states is not ideal in terms of laser applications. What is desired is the highest possible concentration of available states near the bandedge, which results in the lowest necessary injection level to overcome the cavity losses. While quantum wire fabrication presents many more difficulties than the fabrication of quantum wells[4,5], there are also purely theoretical concerns that may reduce expectations for quantum wire laser performance. Non-parabolicities in the bandstructure and heavylight hole mixing affect negatively the threshold current and modulation speed. The efficiency of carrier capture and relaxation mechanisms in quantum wells has been a matter of some concern[6,7]. The situation in quantum wires is even less optimistic, since the absence of intrasubband electron-electron scattering may lead to bottlenecks in the relaxation process due to lack of thermalization in the electron gas which loses energy primarily by emitting monoenergetic LO phonons[8]. In both cases, a detailed numerical simulation is the only solution taking into account the complexity of the underlying mechanisms and the number of interacting particles.

In this paper, we present the results of a detailed theoretical analysis of the optoelectronic properties of the (001) and (111) strained and lattice-matched quantum wire lasers as well as the relaxation characteristics for electrons injected in a thermal distribution at the potential well edge and carry out a thorough comparison of these with similar properties in the quantum well structures. The list of symbols used in this work and of their numerical quantities, where appropriate, is given in Table 1.

The optoelectronic properties of GaAs quantum well and quantum wire lasers can be studied directly on the basis of the bandstructure. The conduction band structure can be computed quite accurately in the effective mass approximation by solving the oneband Schrödinger equation for $s$-type states, while the procedure for solving for the valence band structure is to diagonalize the Kohn-Luttinger four-band Hamiltonian. Both problems can be solved by the finite difference method in which the appropriate derivative terms in the differential equations are approximated as difference terms on a mesh in the wire or well region. The resulting matrix equation is then solved by the standard eigenvalue technique. In Fig. 1 we show the bandstructure and the density of states for the valence band in a $100 \times 100 \AA$ wire. Although the conduction band structure is approximately parabolic, the valence band structure is much more complex owing to the non-parabolicities and heavy-light hole mixing. The effect of the nonparabolicities on the density of states is to broaden or smear the sharp peak at the bandedge which may be expected from the parabolic dispersion analysis. Strain is incorporated in our formalism by calculating 
Table 1. The list of symbols with numerical quantities used in calculations (where appropriate)

\begin{tabular}{|c|c|c|}
\hline Symbol & Physical meaning & Numerical quantity \\
\hline$\ell$ & Lattice mismatch & $-(0.07) \times$ for In, $G a_{1}$, As \\
\hline$n_{0}$ & Refractive index & 3.6 in GaAs \\
\hline$h \omega$ & Photon energy & \\
\hline$A$ & Wire cross-sectional area & \\
\hline$\dot{\epsilon}$ & Light polarization & \\
\hline $\mathbf{P}_{n m}$ & Momentum matrix element & $2 m_{0}\left|\left\langle s\left|p_{1}\right| x\right\rangle\right|^{2}=25.7 \mathrm{ev}$ \\
\hline$f^{\mathrm{c}}\left(E_{n}^{\mathrm{c}}\right), f^{\mathrm{h}}\left(E_{m}^{\mathrm{h}}\right)$ & Electron and hole quasi-fermi functions & \\
\hline ; & Optical confinement per unit width & $0.00025 \square \mathrm{A}$ \\
\hline$w^{\prime}$ & Width in direction of current flow & \\
\hline$L_{\mathrm{c}}$ & Cavity length & $300 \mu \mathrm{m}$ \\
\hline$R$ & Mirror reflectivity & 0.32 \\
\hline$x_{1}$ & Internal cavity loss & $10 \mathrm{~cm}$ \\
\hline$W_{n, n}$ & Scattering rate between states $n$ and $n$ & \\
\hline$M_{n \cdot n}$ & Overlap matrix element between $n$ and $n$ & \\
\hline
\end{tabular}

the splitting between the heavy and light holes[2]. This calculation is based on the assumption of biaxial strain in the growth plane and uniaxial strain in the growth direction. Compressive strain is obtained by adding In to the well region, and the lattice mismatch $\epsilon$ is related to the In mole fraction by $\epsilon=-(0.07) x$. Tensile strain is obtained by adding $P$ to the well region. The resulting bandstructure for the $100 \times 100 \AA \mathrm{In}_{0.2} \mathrm{Ga}_{0.8}$ As wire is shown in Fig. 2. All calculations are done for the same amount of compressive and tensile strain $(|\epsilon|=1.40)$.
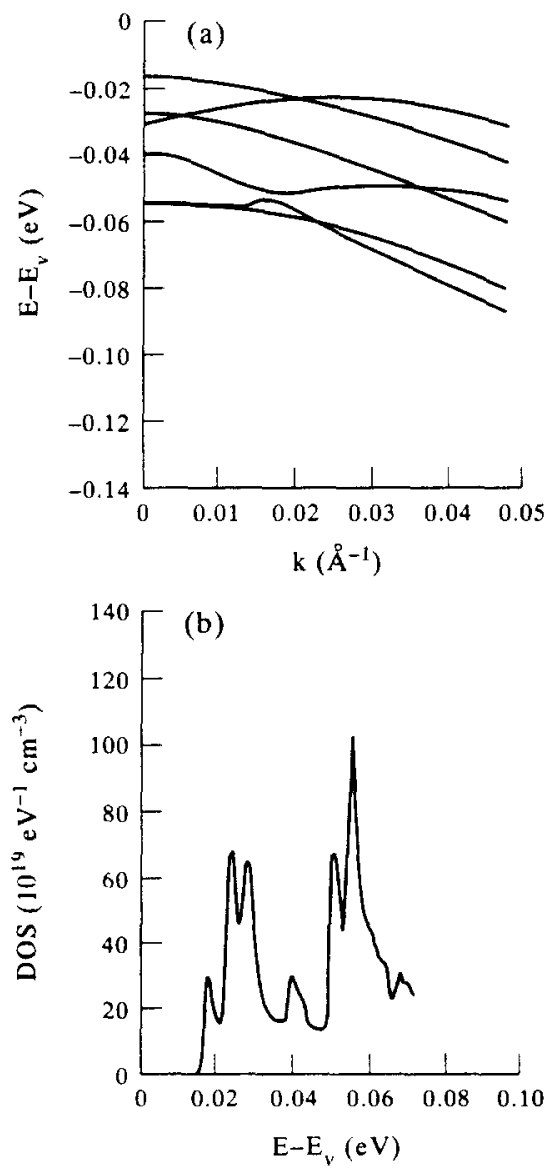

Fig. 1. The valence band structure (a) and density of states (b) for the $100 \times 100 \AA \mathrm{GaAs} / \mathrm{Al}_{0.3} \mathrm{Ga}_{0.7}$ As quantum wire.
The material gain can be calculated from the Fermi Golden Rule yielding (in Gaussian units):

$$
\begin{aligned}
& g(\hbar \omega)=\frac{4 \pi^{2} e^{2} \hbar}{n_{0} c m_{0}^{2} h \omega} \frac{1}{\mathrm{~A}} \frac{2}{2 \pi} \int \mathrm{d} k_{z} \sum_{m, m}\left|\hat{\epsilon} \cdot \mathbf{P}_{n m}\left(k_{z}\right)\right|^{2} \\
& \times \delta\left(E_{n}^{\mathrm{e}}\left(k_{z}\right)-E_{m}^{\mathrm{h}}\left(k_{z}\right)-\hbar \omega\right)\left[f^{\mathrm{e}}\left(E_{n}^{\mathrm{e}}\right)-f^{\mathrm{h}}\left(E_{m}^{\mathrm{h}}\right)\right],
\end{aligned}
$$

where $f^{\mathrm{e}}\left(E_{2 \mathrm{n}}^{\mathrm{c}}\right)$ and $f^{\mathrm{h}}\left(E_{m}^{\mathrm{h}}\right)$ are the electron and hole quasi-Fermi functions respectively. Light experiences optical gain only in the well region; therefore, we characterize the laser optical gain by multiplying the
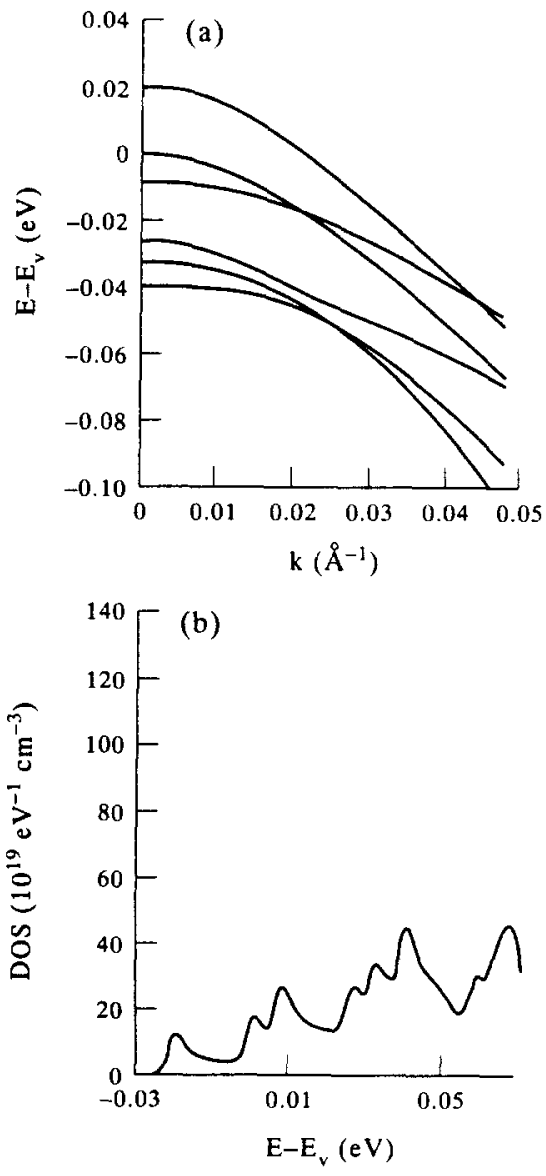

Fig. 2. The valence band structure (a) and density of states (b) for the $100 \times 100 \AA \mathrm{In}_{0.2} \mathrm{Ga}_{0.8} \mathrm{As} / \mathrm{Al}_{0.3} \mathrm{Ga}_{0.7}$ As quantum wire. 
Table 2. The transparency carrier density and the maximum differential gain for a number of quantum wire structures

\begin{tabular}{lrcc}
\hline \multicolumn{2}{c}{ Structure } & Transparency density & Differential gain \\
\hline GaAs (001) & $50 \AA \times 100 \AA$ & $1.10 \times 10^{18} \mathrm{~cm}^{-3}$ & $3.0 \times 10^{-13} \mathrm{~cm}^{2}$ \\
& $100 \AA \times 100 \AA$ & $1.25 \times 10^{18} \mathrm{~cm}^{-3}$ & $2.0 \times 10^{-14} \mathrm{~cm}^{2}$ \\
GaAsP (001) & $50 \AA \times 100 \AA$ & $0.90 \times 10^{18} \mathrm{~cm}^{-3}$ & $5.0 \times 10^{-13} \mathrm{~cm}^{2}$ \\
& $100 \AA \times 100 \AA$ & $1.00 \times 10^{18} \mathrm{~cm}^{-3}$ & $1.2 \times 10^{-13} \mathrm{~cm}^{2}$ \\
InGaAs (001) & $50 \AA \times 100 \AA$ & $0.95 \times 10^{18} \mathrm{~cm}^{-3}$ & $4.7 \times 10^{-13} \mathrm{~cm}^{2}$ \\
& $100 \AA \times 100 \AA$ & $1.05 \times 10^{18} \mathrm{~cm}^{-3}$ & $1.2 \times 10^{-13} \mathrm{~cm}^{2}$ \\
GaAs (11) & $50 \AA \times 100 \AA$ & $0.50 \times 10^{18} \mathrm{~cm}^{-3}$ & $7.0 \times 10^{-13} \mathrm{~cm}^{2}$ \\
& $100 \AA \times 100 \AA$ & $0.60 \times 10^{18} \mathrm{~cm}^{-3}$ & $4.5 \times 10^{-13} \mathrm{~cm}^{2}$ \\
\hline
\end{tabular}

material gain by an optical confinement factor $\Gamma=\gamma W$, where $\gamma$ is the optical confinement per unit width and $W$ is the width of the quantum wire in the direction of the current flow.

The differential gain is given by the partial derivative of the material gain with respect to the carrier concentration. The recombination rate is also necessary in order to determine the threshold current and is given by the dipole transition rate for carrierconfined states:

$$
\begin{gathered}
R_{\mathrm{sp}}=\int \mathrm{d}(h \omega) \frac{4 e^{2} n_{0} \hbar \omega}{3 m_{0}^{2} c^{3} \hbar^{2}} \frac{2}{2 \pi} \int \mathrm{d} k_{z} \sum_{n, m}\left|\mathbf{P}_{n m}\left(k_{z}\right)\right|^{2} \delta \\
\times\left(E_{n}^{\mathrm{e}}\left(k_{z}\right)-E_{m}^{\mathrm{h}}\left(k_{z}\right)-\hbar \omega\right)\left[f^{\mathrm{e}}\left(E_{n}^{\mathrm{c}}\right)\right]\left[1-f^{\mathrm{h}}\left(E_{m}^{\mathrm{h}}\right)\right] .
\end{gathered}
$$

Note that the above expressions are applicable to quantum wires. The formalism for quantum wells can be found in Ref.[3]. In order to provide a theoretical estimate of the threshold carrier density, we set up the optical loop gain for the cavity losses, resulting in the transparency condition:

$$
\Gamma g\left(n_{\mathrm{th}}, E_{\text {peak }}\right)=\frac{1}{L_{\mathrm{C}}} \ln \frac{1}{R}+\alpha_{\mathrm{i}},
$$

where $g\left(n_{\mathrm{th}}, E_{\text {peak }}\right)$ is the material gain at the peak mode $E_{\text {peak }}, L_{\mathrm{c}}$ is the length of the cavity, $R$ is the reflectivity of the mirrors, and $\alpha_{i}$ is the intrinsic cavity loss. We assume a total loss of $50 \mathrm{~cm}^{-1}$ for the Fabry-Perot laser cavity. Below threshold, the current is completely due to spontaneous recombination, and the threshold current density is $J_{\mathrm{th}}=e R_{\mathrm{sp}}\left(n_{\mathrm{th}}\right)$.

The transparency carrier density and maximum differential gain are shown for a number of latticematched and strained wires in Table 2. The same parameters are shown in Table 3 for several optimized quantum well structures. Reduction in the transparency density can be achieved by compressive strain as well as tensile strain (for a large enough fraction of $P$ in the active region, as assumed here), or orienting the wire axis along the [111] direction. Growth along [111] offers a more than $50 \%$ decrease in the transparency density due to the greater separation between the subbands resulting in a more nearly parabolic dispersion. The effect of strain is similar, but less pronounced (see Fig. 2). The transparency densities in quantum wells are $30-40 \%$ greater than those in quantum wires for optimized wire and well sizes. A lower transparency density normally corresponds to a lower threshold current for identical optical cavities provided the recombination rates are comparable for different structures. We have calculated a threshold current density of $80 \mathrm{~A} / \mathrm{cm}^{2}$ for the $100 \times 100 \AA \mathrm{GaAs}$ wire, and that of $30 \mathrm{~A} / \mathrm{cm}^{2}$ for the $\mathrm{In}_{0.2} \mathrm{Ga}_{0.8}$ As wire of the same cross section. This corresponds to a threshold density of $560 \mathrm{~A} / \mathrm{cm}^{2}$ for the $50 \AA \mathrm{GaAs}$ well and $150 \mathrm{~A} / \mathrm{cm}^{2}$ for the $50 \AA$ $\mathrm{In}_{0.2} \mathrm{Ga}_{0.8}$ As well. While the threshold currents are found to be much smaller in quantum wires than in quantum wells, we note that the reduction achieved by incorporation of strain is not as drastic.

The gain spectrum found as a result of our calculations is much more narrow than that calculated for quantum well lasers explicable by the sharpness and narrowness in the density of states function in a quantum wire. The narrow gain spectrum not only insures excellent mode selectivity and ease of singlemode operation at low injected currents, but also provides efficient damping of the relaxation oscillations observed as the response to a step in the driving current since the sidemode power is very low. Also shown in Tables 2 and 3 is the maximum differential gain achievable in each structure. The differential gain is an important parameter for high speed modulation of semiconductor lasers. It is clear that quantum wire lasers are capable of significantly higher differential gains than quantum wells. in some cases of the order of $10^{-13} \mathrm{~cm}^{2}$. Higher differential gains may lead to a much lower linewidth of emission in quantum wires. However, high speed modulation in quantum wires may be degraded by the extremely slow relaxation processes as discussed in the following section.

The modulation speed for a semiconductor laser is normally calculated with the assumption of a quasiFermi distribution for both electrons and holes. This assumption is excellent for bulk lasers, in which the

Table 3. The transparency carrier density and the maximum differential gain for a number of quantum well structures

\begin{tabular}{lcc}
\hline Structure & Transparency density & Differential gain \\
\hline $50 \AA$ GaAs $(001)$ & $1.80 \times 10^{18} \mathrm{~cm}^{-3}$ & $3.0 \times 10^{-14} \mathrm{~cm}^{2}$ \\
$50 \AA$ InGaAs $(001)$ & $1.25 \times 10^{18} \mathrm{~cm}^{-3}$ & $1.0 \times 10^{-14} \mathrm{~cm}^{2}$ \\
$50 \AA$ GaAs $(111)$ & $0.75 \times 10^{18} \mathrm{~cm}^{-3}$ & $2.0 \times 10^{-14} \mathrm{~cm}^{2}$ \\
\hline
\end{tabular}


intraband relaxation times are of the order of $1 \mathrm{ps}$. In quantum wells. however, the relaxation time increases by as much as an order of magnitude due to the reduction in the momentum space and difficulty in coupling of the extended states in the barrier to the confined states in the well. While the capture time in quantum wells depends on width[7] and structure, the relaxation time for electrons injected into the well is of the order of picoseconds. The relaxation time for holes is expected to be much lower than that for electrons primarily due to the nigher density of states and a greater number of subband levels in the valence band.

In quasi-one-dimensional systems, the only intrasubband electron-electron scattering process allowed by the requirement of simultaneous conservation of energy and momentum is an exchange of states by the colliding electrons. The main mechanism for energy loss in quantum wires is expected to be emission of polar optical phonons (POP) whose energy is nearly constant near the zone center. The lack of randomization by electron-electron scattering may result in bottlenecks in the relaxation process, in which the temperature of the electron distribution significantly exceeds that of the lattice on a time scale of $100 \mathrm{ps}$. The bottlenecks are eventually overcome owing to the small, yet finite, inelasticity of the acoustic phonons (AP) and carrier-carrier scattering processes, but the relaxation times may range, depending on the wire size, from $10 \mathrm{ps}$ to several hundred ps[9]. With such long relaxation times, the assumption of the FermiDirac distribution for electrons in the small-signal analysis is no longer correct. In fact, laser modulation with frequencies exceeding the reciprocal of the relaxation time is problematic. In this sense, we say that electron relaxation sets the upper limit on the laser modulation frequency.

We calculate the electron relaxation times for a number of quantum wire cross sections by an ensemble Monte Carlo simulation. A large number of electrons $(5000)$ are injected in a thermal distribution at the edge of the potential well formed by the barrier region. They are allowed to interact with the reservoir of acoustic and polar optical phonons, as well as between themselves, and their progress is recorded as a function of time. The scattering rates are calculated from the Fermi Golden Rule. Since we consider quantum wires of cross sections from $100 \times 100 \AA$ up, we assume that the coupling between the confined electronic states and localized interface phonons is significantly smaller than that between electrons and confined bulklike phonons, and perform our calculations only for the latter interaction. The Fröhlich coupling between polar optical phonons and electrons is much stronger than the deformation-potential coupling between acoustic phonons, the POP scattering rates are on the order of $10^{12} \mathrm{~s}^{-1}$ with sharp peaks corresponding to the peaks in the density of states, while the AP scattering rates are smaller by an order of magnitude. The effect of screening is incor-

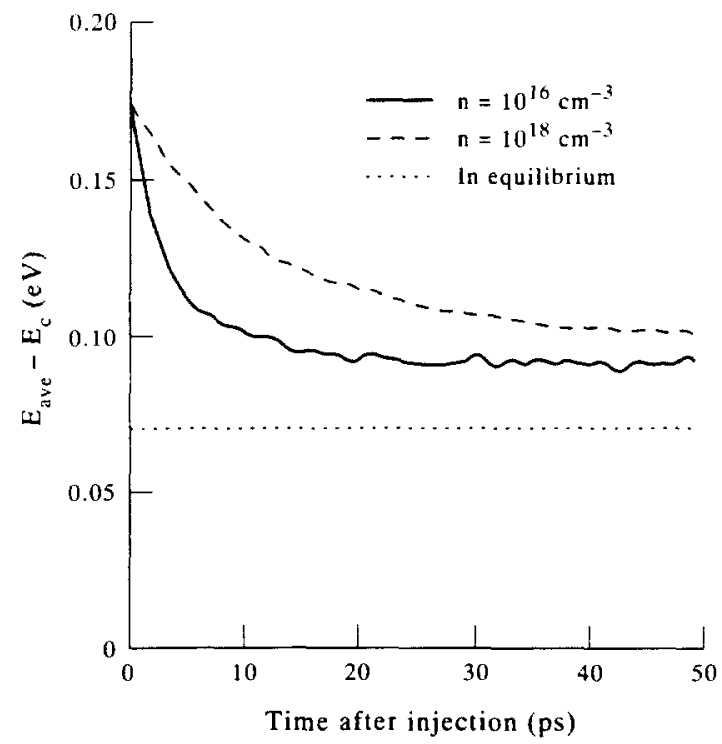

Fig. 3. The energy averaged over a large number of electrons as a function of time after injection for a $100 \times 100 \AA \mathrm{GaAs}$ quantum wire for low and high injected carrier concentrations. The horizontal line corresponds to the expectation value of the energy for the equilibrium Fermi distribution.

porated using the Thomas-Fermi theory. The details of the formalism are presented elsewhere[10].

In Fig. 3, we plot the electron relaxation times for two electron concentrations for the $100 \times 100 \AA$ quantum wire. At a high screening carrier density characteristic of laser operation, the relaxation time ranges from $120 \mathrm{ps}$ for the $100 \times 100 \AA$ wire to $30 \mathrm{ps}$ for the $200 \times 200 \AA$ wire (Fig. 4). However, for large wire sizes, the threshold current approaches that in quantum wells; therefore, we must conclude that

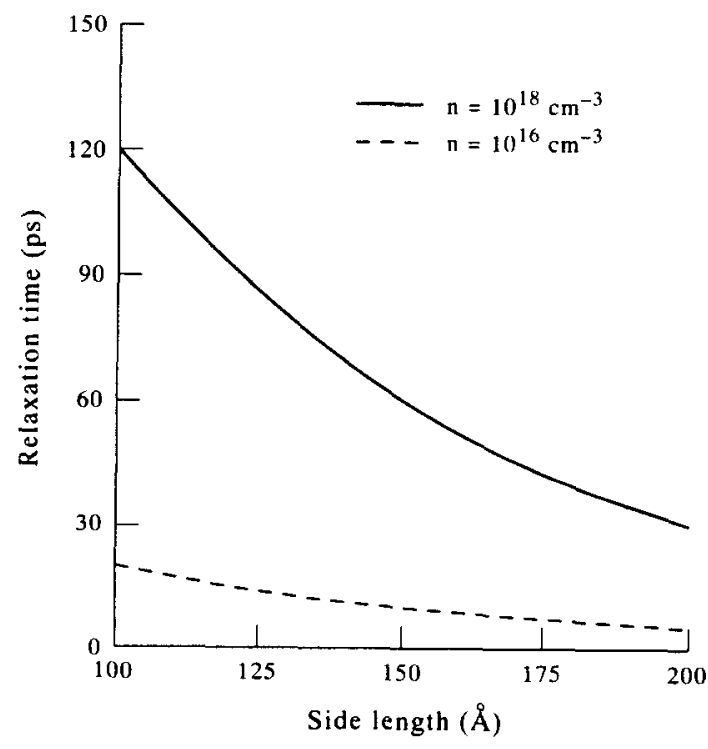

Fig. 4. The electron relaxation time as a function of the wire cross section. A square cross section is considered, the length of the side is given for carrier concentrations of $10^{16}$ and $10^{18} \mathrm{~cm}^{-3}$. 
based on the model described above, the relaxation times in quantum wires exceed those in quantum wells by at least an order of magnitude. The theoretical limit on the modulation frequency for $100 \times 100 \AA$ quantum wire laser is thus calculated to be less than $10 \mathrm{GHz}$. That is well below the highest modulation frequencies obtained for some quantum well lasers.

We have made a comparative analysis of the optoelectronic properties in lattice-matched and strained quantum well and quantum wire systems. We find that in quantum wires the transparency carrier density is reduced considerably in comparison with quantum wells, which leads to significantly lower threshold currents. The maximum differential gain is increased in quantum wires by an order of magnitude in comparison with quantum wells, which may lead to a lower linewidth for emission. However, consideration of the relaxation processes with the inclusion of electron-phonon and electron-electron interaction leads us to believe that the electron relaxation time in quantum wires may be increased to over $100 \mathrm{ps}$ and may constitute the upper limit on the modulation frequency. Therefore, although the quan- tum wire laser requires a significantly lower threshold current than a comparable quantum well laser, high speed operation in quantum wire lasers may be degraded in comparison with quantum wells due to exceedingly slow carrier relaxation processes.

\section{REFERENCES}

1. Y. Arakawa and A. Yariv, IEEE J. Quantum Electron 22, 1887 (1986).

2. J. P. Loehr and J. Singh, IEEE J. Quantum Electron. 27, $708(1991)$

3. Y. Lam, J. P. Loehr and J. Singh, IEEE J. Quantum Electron. 28, 1248 (1992).

4. P. M. Petroff, A. C. Gossard, R. A. Logan and W. Wiegman, Appl. Phys. Lett. 41, 635 (1982)

5. M. Tsuchiya, J. M. Gaines, R. H. Yan, R. J. Simes, P. O. Holtz, L. A. Colden and P. M. Petroff, Phys. Rev. Lett. 62, 466 (1989).

6. S. M. Goodnick and P. Lugli, Phys. Rer. B 37, 2578 (1988).

7. J. A. Brum and G. Bastard, Phys. Rev. B 33, 1420 (1986).

8. J. P. Leburton, Phys. Rev. B 45, 11022 (1992)

9. I. Vurgaftman and J. Singh, Appl. Phys. Lett. 62, 2251 (1993).

10. I. Vurgaftman and J. Singh, submitted for publication. 